\title{
Measuring the Intra-Atomic Exchange Energy in Rare-Earth Adatoms
}

\author{
Marina Pivetta $\odot,{ }^{1}$ François Patthey, ${ }^{1}$ Igor Di Marco $\odot,{ }^{2,3,4}$ Arya Subramonian, ${ }^{2,5}$ Olle Eriksson,,${ }^{3,6}$ \\ Stefano Rusponi $\odot^{1}$, and Harald Brune $\odot^{1}$ \\ ${ }^{1}$ Institute of Physics, Ecole Polytechnique Fédérale de Lausanne, CH-1015 Lausanne, Switzerland \\ ${ }^{2}$ Asia Pacific Center for Theoretical Physics, Pohang, 37673, Korea \\ ${ }^{3}$ Department of Physics and Astronomy, Uppsala University, Box 516, SE-75120, Uppsala, Sweden \\ ${ }^{4}$ Department of Physics, POSTECH, Pohang, 37673, Korea \\ ${ }^{5}$ Government Polytechnic College, Kaduthuruthy, Kerala, India 686604 \\ ${ }^{6}$ School of Science and Technology, Örebro University, SE-70182 Örebro, Sweden
}

(Received 29 January 2020; revised 5 June 2020; accepted 23 July 2020; published 9 September 2020)

\begin{abstract}
We present the first experimental determination of the intra-atomic exchange energy between the inner $4 f$ and the outer $6 s 5 d$ shells in rare-earth elements. Inelastic electron tunneling spectroscopy on individual rare-earth atoms adsorbed on metal-supported graphene reveals an element-dependent excitation, with energy between 30 and $170 \mathrm{meV}$, linearly increasing with the spin angular momentum of the $4 f$ shell. This observation is possible owing to the strong spin polarization of the outer shells, characteristic of rare-earth adatoms on graphene. This polarization gives rise to a giant magnetoresistance of up to $75 \%$ observed for Dy on graphene/ $\operatorname{Ir}(111)$ single-atom magnets. Density functional theory calculations of the $6 s 5 d$ shell spin polarizations and of their intra-atomic exchange constants with the $4 f$ shell yield exchange energies in agreement with the experimental values. These results prove that the description of the spin dynamics in RE considering only the $4 f-5 d$ interaction is oversimplified. A more realistic treatment requires us to consider a multishell intra-atomic exchange in which both $6 s$ and $5 d$ shells are taken into account, with the $4 f-6 s$ contribution possibly prevailing over the $4 f-5 d$ one. Our findings are important for the general understanding of magnetism in rare earths, whether they are in bulk compounds or as surface adsorbed atoms and clusters. The results presented here also push for a revision of the description of the spin dynamics in rare-earth-based systems.
\end{abstract}

DOI: $10.1103 /$ PhysRevX.10.031054

Subject Areas: Condensed Matter Physics, Magnetism, Quantum Physics

\section{INTRODUCTION}

Magnetic systems represent one of the fastest-moving frontiers in solid-state physics and materials science. Recent advancements have shown that spintronics and quantum computation require strongly correlated systems in which strong spin-orbit coupling, large crystal fields, and high exchange energies come into play. Because of their high spin-orbit coupling, rare-earth (RE) elements are hence key ingredients for future applications in these fields [1-3].

The intra-atomic exchange interaction, in particular, has a strong effect on the static and dynamic magnetic properties of systems containing RE species. A well-known example is represented by RE-transition metal (TM) permanent magnets such as $\mathrm{SmCo}_{5}$ or $\mathrm{Nd}_{2} \mathrm{Fe}_{14} \mathrm{~B}$. In these systems, the direct magnetic coupling between the $4 f$ orbitals of the

Published by the American Physical Society under the terms of the Creative Commons Attribution 4.0 International license. Further distribution of this work must maintain attribution to the author(s) and the published article's title, journal citation, and DOI.
$\mathrm{RE}$, bearing most of the atom magnetic moment, with the $3 d$ shell of TM is negligible because of the strongly localized nature of the $4 f$ electrons. Instead, the $3 d-4 f$ interaction is mediated by the moment of the external $6 s 5 d$ shells of the RE, which is exchange coupled with both the $3 d$ moment of the TM atoms (interatomic exchange) and the $4 f$ moment of the RE itself (intra-atomic exchange) [4-6].

Recently, several investigations on elemental [7-12] and alloyed [13-15] RE systems have highlighted the importance of the intra-atomic exchange coupling also for dynamic magnetic properties. For example, different dynamics at the ps scale have been reported for the $5 d$ and $4 f$ magnetic moments in $\mathrm{Gd}$, demonstrating the breakdown of the intra-atomic exchange coupling at very short timescales [10]. Ultimately, understanding the spin dynamics in magnetic materials is key to reaching high-speed writing and reading in spintronics and magnetic recording.

Despite its importance, so far the intra-atomic exchange energy could not be accessed experimentally. Consequently, the description of the magnetism of all the above-mentioned systems was exclusively based on calculated exchange energies [16-19]. In solids and clusters, the measurement 
(a)

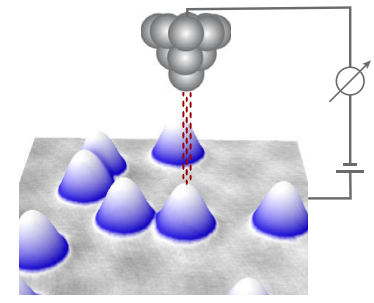

(c)

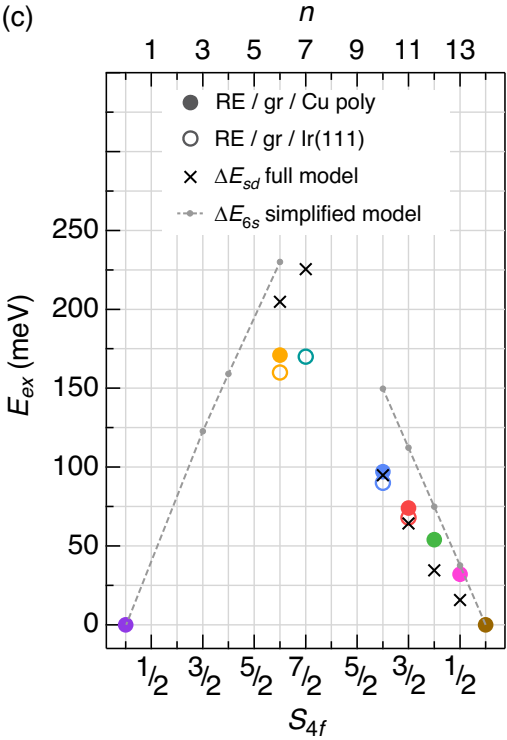

(b)

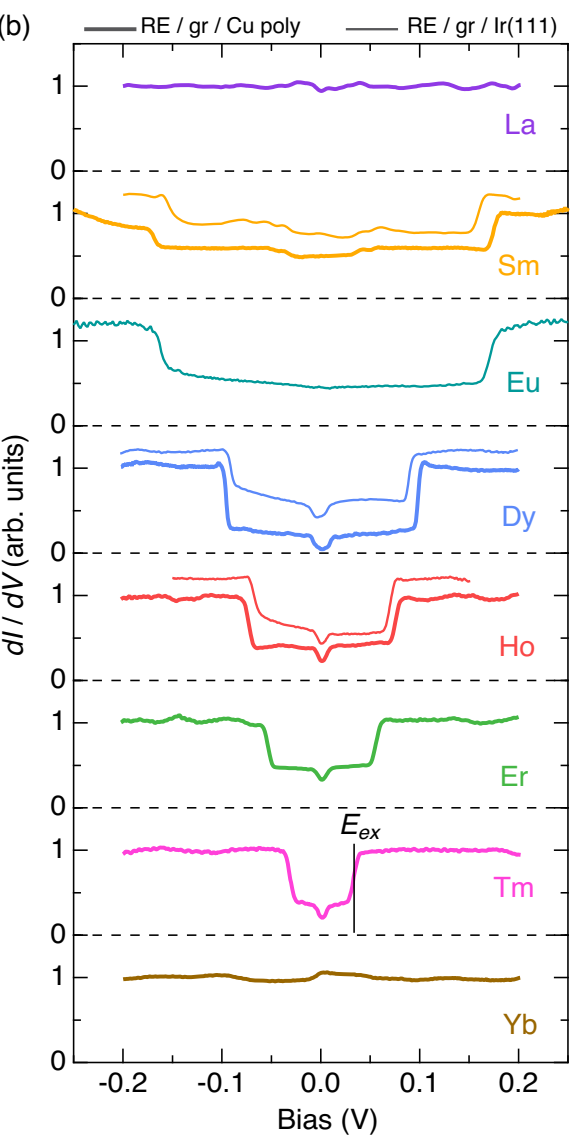

FIG. 1. IETS spin excitations originating from breaking the intra-atomic exchange interaction. (a) 3D rendering of a constant current STM image of Dy atoms on $\operatorname{gr} / \operatorname{Ir}(111)\left(V_{\mathrm{t}}=-0.5 \mathrm{~V}, I_{\mathrm{t}}=30 \mathrm{pA}, T=6.5 \mathrm{~K}\right)$, together with a sketch illustrating the STM measurements. (b) Conductance spectra acquired on $\mathrm{La}, \mathrm{Sm}, \mathrm{Eu}, \mathrm{Dy}, \mathrm{Ho}, \mathrm{Er}, \mathrm{Tm}$, and $\mathrm{Yb}$ single atoms adsorbed on gr/polycrystalline copper (thick lines) and on $\mathrm{gr} / \operatorname{Ir}(111)$ (thin lines). The IETS excitation corresponding to breaking the intra-atomic exchange is labeled $E_{\text {ex }}$ on the Tm spectrum as an example. Each spectrum is the average of about ten spectra acquired on different atoms. Spectra are background corrected and normalized to 1 . Spectra on $\mathrm{gr} / \operatorname{Ir}(111)$ are vertically offset by 0.3 for clarity. Spectroscopy parameters: $I_{\text {set }}=200 \mathrm{pA}, V_{\text {set }}=-0.2 \mathrm{~V}$ for all spectra except for $\mathrm{Sm}$ on $\mathrm{gr} / \mathrm{Cu}$ and $\mathrm{Eu}$ on gr $/ \mathrm{Ir}\left(V_{\text {set }}=-0.25 \mathrm{~V}\right)$, and for Ho on gr $/ \mathrm{Ir}$ $\left(V_{\text {set }}=-0.15 \mathrm{~V}\right), V_{\text {mod }}=5 \mathrm{mV}$ peak-to-peak, $T=6.5 \mathrm{~K}$. All spectra are acquired using a W tip. (c) $E_{\text {ex }}$ vs $S_{4 f}$, the spin angular momentum of the $4 f$ shell, and $n$, the number of $f$ electrons. The error bar is of the order of $\pm 1 \mathrm{meV}$, i.e., smaller than the size of the symbols, and corresponds to the width of the observed energy distribution. Calculated values of the excitation energy $\Delta E$ obtained with simplified and full models are also reported (see text).

of this quantity is hampered by the concomitant effect of several interactions such as interatomic and intra-atomic exchange, crystal field, and spin-orbit coupling. Single atoms adsorbed on a surface are simplified model systems, enabling us to disentangle these interactions. Scanning tunneling microscopy (STM) and inelastic electron tunneling spectroscopy (IETS) are ideal to study magnetic excitations on individual atoms since the tunneling electrons can exchange momentum with the spin localized on the adatom. In the past, this technique allowed one to investigate the energy spectrum generated by the combined effect of crystal field and Zeeman interaction on TM adatoms [20-23].

Here, we use IETS on RE adatoms on graphene to measure the intra-atomic exchange interaction between $4 f$ and $6 s 5 d$ electrons. The absence of interatomic exchange with surrounding atoms and the electronic configuration proper to $\mathrm{RE}$ adatoms on graphene makes these adatom-substrate combinations ideal for such an investigation. Measurements on several RE elements show that the observed IETS excitation energies $E_{\text {ex }}$ are proportional to the spin angular momentum $S_{4 f}$, as expected for the intraatomic exchange interaction, and their values are in the predicted energy range. Moreover, our data imply a strong spin polarization of the $6 s 5 d$ outer shells, as also demonstrated by the giant magnetoresistance observed in spinpolarized STM measurements for the Dy adatom, the only RE element reported to show long spin lifetime on this substrate $[24,25]$. Density functional theory (DFT) calculations of the outer-shell polarizations and of the intra-atomic exchange integrals yield exchange energies in agreement with the experimental values. 
TABLE I. Comparison between experimental and calculated intra-atomic exchange energies. We show the experimental $E_{\text {ex }}$ from IETS spectra for RE on gr/Cu and gr/ $\operatorname{Ir}(111)$. Simplified model: $\Delta E=\Delta E_{6 s}=2 J_{4 f-6 s} \Delta S_{6 s} S_{4 f}$, with $J_{4 f-6 s}$ from Ref. [18] and electronic configuration $6 s^{1} 5 d^{0} 4 f^{n}$, with a fully spin-polarized $6 s$ shell $\left(\Delta S_{6 s}=2 S_{6 s}=1\right)$. Full model: $\Delta E=\Delta E_{s d}=2\left(J_{4 f-6 s} \Delta S_{6 s}+\right.$ $\left.J_{4 f-5 d} \Delta S_{5 d}\right) S_{4 f}$, with $J_{4 f-6 s}, J_{4 f-5 d}$, and spins $\left(S_{6 s}, S_{5 d}\right)$ from our DFT calculations $\left(\Delta S_{6 s}=2 S_{6 s}, \Delta S_{5 d}=2 S_{5 d}\right)$. Note that $S_{4 f}$ values are calculated according to Hund's first rule in both models. La and $\mathrm{Yb}\left(S_{4 f}=0\right)$ are not included. All energies are in meV.

\begin{tabular}{|c|c|c|c|c|c|c|c|c|}
\hline $\mathrm{RE}$ & & $S_{4 f}$ & $S_{6 s}$ & $S_{5 d}$ & $J_{4 f-6 s}$ & $J_{4 f-5 d}$ & $E_{\mathrm{ex}}$ & $\Delta E$ \\
\hline \multirow[t]{4}{*}{$\mathrm{Sm}$} & $\mathrm{gr} / \mathrm{Cu}$ & $\cdots$ & $\cdots$ & $\cdots$ & $\cdots$ & $\cdots$ & 171 & $\cdots$ \\
\hline & $\mathrm{gr} / \operatorname{Ir}(111)$ & $\cdots$ & $\cdots$ & $\cdots$ & $\cdots$ & $\cdots$ & 160 & $\cdots$ \\
\hline & Simplified model $\left(\Delta E_{6 s}\right)$ & 3 & 0.5 & $\cdots$ & 38.3 & $\cdots$ & $\cdots$ & 229.8 \\
\hline & Full model $\left(\Delta E_{s d}\right)$ & 3 & 0.22 & 0.08 & 40 & 104 & $\cdots$ & 205.4 \\
\hline \multirow[t]{2}{*}{$\mathrm{Eu}$} & $\operatorname{gr} / \operatorname{Ir}(111)$ & $\ldots$ & $\cdots$ & $\cdots$ & $\cdots$ & $\cdots$ & 170 & $\ldots$ \\
\hline & Full model $\left(\Delta E_{s d}\right)$ & $7 / 2$ & 0.23 & 0.07 & 39 & 99 & $\cdots$ & 222.6 \\
\hline \multirow[t]{4}{*}{ Dy } & $\mathrm{gr} / \mathrm{Cu}$ & $\cdots$ & $\cdots$ & $\cdots$ & $\cdots$ & $\cdots$ & 97 & $\cdots$ \\
\hline & $\operatorname{gr} / \operatorname{Ir}(111)$ & $\cdots$ & $\cdots$ & $\cdots$ & $\cdots$ & $\cdots$ & 90 & $\cdots$ \\
\hline & Simplified model $\left(\Delta E_{6 s}\right)$ & 2 & 0.5 & $\cdots$ & 37.4 & $\cdots$ & $\cdots$ & 149.6 \\
\hline & Full model $\left(\Delta E_{s d}\right)$ & 2 & 0.23 & 0.04 & 37 & 85 & $\cdots$ & 95.3 \\
\hline \multirow[t]{4}{*}{ Ho } & $\mathrm{gr} / \mathrm{Cu}$ & $\ldots$ & $\cdots$ & $\cdots$ & $\cdots$ & $\cdots$ & 74 & $\cdots$ \\
\hline & $\mathrm{gr} / \operatorname{Ir}(111)$ & $\cdots$ & $\cdots$ & $\cdots$ & $\cdots$ & $\cdots$ & 68 & $\cdots$ \\
\hline & Simplified model $\left(\Delta E_{6 s}\right)$ & $3 / 2$ & 0.5 & $\cdots$ & 37.4 & $\cdots$ & $\cdots$ & 112.2 \\
\hline & Full model $\left(\Delta E_{s d}\right)$ & $3 / 2$ & 0.21 & 0.03 & 37 & 81 & $\cdots$ & 61.2 \\
\hline \multirow[t]{3}{*}{ Er } & $\mathrm{gr} / \mathrm{Cu}$ & $\cdots$ & $\cdots$ & $\cdots$ & $\cdots$ & & 54 & $\cdots$ \\
\hline & Simplified model $\left(\Delta E_{6 s}\right)$ & 1 & 0.5 & $\cdots$ & 37.4 & & $\cdots$ & 74.8 \\
\hline & Full model $\left(\Delta E_{s d}\right)$ & 1 & 0.19 & 0.02 & 37 & 76 & $\cdots$ & 34.2 \\
\hline \multirow[t]{3}{*}{$\mathrm{Tm}$} & $\mathrm{gr} / \mathrm{Cu}$ & $\cdots$ & $\ldots$ & $\cdots$ & $\cdots$ & $\cdots$ & 32 & $\cdots$ \\
\hline & Simplified model $\left(\Delta E_{6 s}\right)$ & $1 / 2$ & 0.5 & $\cdots$ & 37.6 & $\cdots$ & $\cdots$ & 37.6 \\
\hline & Full model $\left(\Delta E_{s d}\right)$ & $1 / 2$ & 0.18 & 0.02 & 36 & 72 & $\ldots$ & 15.8 \\
\hline
\end{tabular}

\section{RESULTS AND DISCUSSION}

We carried out STM and IETS measurements on RE adatoms on graphene (gr) grown on both $\operatorname{Ir}(111)$ and polycrystalline $\mathrm{Cu}$; see Fig. 1(a). The typical adatom coverage was less than $0.5 \%$ of a monolayer (ML), with $1 \mathrm{ML}$ defined as one adatom per graphene unit cell; see the Appendixes $\mathrm{A}$ and $\mathrm{C}$ for more details about sample preparation and characterization. Figure 1(b) shows differential conductance $(d I / d V)$ spectra for eight RE species adsorbed on $\mathrm{gr} / \mathrm{Cu}$ and on $\mathrm{gr} / \mathrm{Ir}(111)$. For six of them $(\mathrm{Sm}$, $\mathrm{Eu}, \mathrm{Dy}, \mathrm{Ho}, \mathrm{Er}$, and Tm), we observe prominent inelastic excitations at energy $\pm E_{\text {ex }}$ (marked on the Tm spectrum as an example) that depend on the RE element, while no inelastic features are observed for $\mathrm{La}$ and $\mathrm{Yb}$.

The $E_{\mathrm{ex}}$ values are reported in Fig. 1(c), with full symbols for $\mathrm{gr} / \mathrm{Cu}$ and with open symbols for $\mathrm{gr} / \mathrm{Ir}(111)$, as a function of $S_{4 f}$, the $4 f$ spin angular momentum calculated via Hund's first rule, and of $n$, the number of $f$ electrons for the RE atoms in the gas phase. For the RE elements measured on both supporting substrates (Sm, Dy, and Ho), the $E_{\text {ex }}$ values are $10 \%$ lower on $\operatorname{gr} / \operatorname{Ir}(111)$ than on $\mathrm{gr} / \mathrm{Cu}$. No significative influence of the $\mathrm{gr} / \operatorname{Ir}(111)$ moiré pattern on the inelastic excitations is observed. Concerning the polycrystalline copper substrate, the surface is inhomogeneous owing to the existence of microcrystals with different terminations. However, on each $\mathrm{Cu}$ microcrystal, the spectra are equivalent (i.e., for a given RE species, the differences in the inelastic step energy are in the error bar of $\pm 1 \mathrm{meV}$ ), and the deviations in $E_{\mathrm{ex}}$ over the entire sample are smaller than $10 \%$. The spectra reported in Fig. 1(b) and the corresponding $E_{\text {ex }}$ values in Fig. 1(c) are representative of the most frequent ones. From Fig. 1(c), the proportionality between $E_{\text {ex }}$ and $S_{4 f}$ is evident on both supporting substrates. All the $E_{\mathrm{ex}}$ values are also summarized in Table I.

To rationalize this observation, we have to consider the electronic and spin configuration of these adatoms. In the gas phase, RE atoms are divalent $\left(6 s^{2} 5 d^{0} 4 f^{n}\right)$, as schematically shown in Fig. 2(a), a configuration in which there is no magnetic moment associated with the outer-shell electrons. Upon adsorption on a surface, depending on the competition between atomic correction energy and surface binding energy [26], both divalent [27] and trivalent $\left(6 s^{2} 5 d^{1} 4 f^{n-1}\right)[27,28]$ electronic configurations have been reported. In particular, on graphene, the RE adatom forms an ioniclike bond with the $\mathrm{C}$ atoms, resulting in a charge transfer of about one electron from the atom to graphene [29-31]. To a first approximation, this charge transfer 
(a)
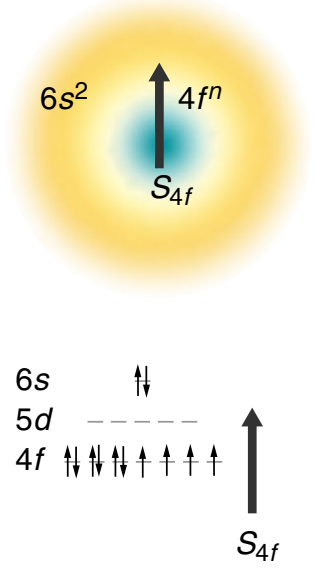

(b)
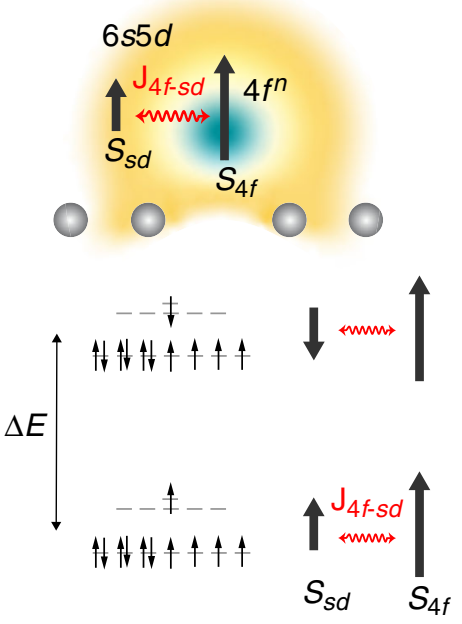

FIG. 2. Intra-atomic exchange in RE atoms. (a) Top diagram: Sketch of the radial distribution of the $4 f$ and $6 s$ atomic orbitals for a gas phase RE atom $\left(6 s^{2} 5 d^{0} 4 f^{n}\right)$. Bottom diagram: Corresponding atomic shell filling (case of Dy, $n=10$ ). (b) Top diagram: Adsorption on graphene, promoting the transfer of approximately one electron to graphene, resulting in partially filled, spin-polarized outer shells $\left[(6 s 5 d)^{1} 4 f^{n}\right]$. Note that $J_{4 f-s d}$ is the intra-atomic exchange constant. Bottom diagram: Exchange-split energy levels for the case of an outer shell with spin $S_{s d}=1 / 2$. The ferromagnetic and antiferromagnetic configurations are separated by $\Delta E$.

leaves the adatom in an electronic configuration close to $6 s^{1} 5 d^{0} 4 f^{n}$ or to $6 s^{1} 5 d^{1} 4 f^{n-1}$, depending on the tendency of each RE species to be divalent or trivalent.

Owing to the exchange coupling between the $4 f$ and $6 s 5 d$ electrons, the partially filled $6 s 5 d$ outer shells become spin polarized [32-34], with spin angular moment $S_{s d}$. The intra-atomic exchange interaction between the spins of inner and outer shells can be divided into isotropic (spinspin) and anisotropic contributions. Because the isotropic term is dominating over the anisotropic ones [18,35], it is a good approximation to write the exchange Hamiltonian as $H_{4 f-s d}=-2 J_{4 f-s d} \mathbf{S}_{s d} \cdot \mathbf{S}_{4 f}[4,5,16]$, where a positive value of the exchange constant $J_{4 f-s d}$ favors a parallel alignment of the two spin moments with respect to the antiparallel configuration. The energy difference between parallel and antiparallel coupling is $\Delta E$; see Fig. 2(b). This formulation is equivalent to the one commonly used to describe the exchange interaction in RE compounds, where the spin $\mathbf{S}_{4 f}$ is replaced by $\left(g_{J}-1\right) \mathbf{J}_{4 f}$, with $g_{J}$ the Landé $g$ factor and $\mathbf{J}_{4 f}$ the total angular moment, leading to the wellknown dependence of the Curie temperature on the de Gennes factor [32,36-39].

Except for La, the graphene-adsorbed RE species reported in Fig. 1(c) possess a $4 f^{n}$ filling of the shell, as expected from calculation of the total atomic correction energy [26] and confirmed by our DFT calculations (see

Appendix B). These results are also in agreement with $\mathrm{x}$-ray absorption spectroscopy measurements for Dy, Ho, and $\mathrm{Er}$ on graphene $/ \operatorname{Ir}(111)$ [25]. In a simplified model, the electronic configuration of these atoms is $6 s^{1} 5 d^{0} 4 f^{n}$, with a fully spin-polarized $6 s$ shell, meaning that $S_{s d}=S_{6 s}=1 / 2$. Thus, the reversal of the $S_{6 s}$ spin $\left(\Delta S_{6 s}=1\right)$ costs $\Delta E_{6 s}=2 J_{4 f-6 s} S_{4 f}$, as sketched in Fig. 2(b).

In IETS measurements, the conservation of spin angular momentum imposes selection rules for singleelectron excitation processes, which can only change the localized spin by $0, \pm 1$. Thus, a tunneling electron having sufficient energy (i.e., $\Delta E_{6 s}$ ) can transfer its spin moment to the $6 s$ electrons, breaking the ferromagnetic coupling between the inner and outer shells and inducing the $S_{6 s}$ reversal.

Assuming the values obtained from Hund's first rule for $S_{4 f}[4,5]$ and using the calculated values of $J_{4 f-6 s}$ reported in the literature [18], we obtain $\Delta E_{6 s}$ values that have the correct order of magnitude and qualitatively reproduce the experimental trend [see Fig. 1(c), grey line, and Table I, simplified model]. Both La (empty $4 f$ shell) and $\mathrm{Yb}$ (full $4 f$ shell) have $S_{4 f}=0$, and no inelastic features are expected, in agreement with the experiment. The good correspondence between the results of the model and observed values allows us to unambiguously identify $E_{\mathrm{ex}}$ as the energy required to change the alignment between $S_{4 f}$ and $S_{s d}$ from parallel to antiparallel, i.e., to break the intraatomic exchange coupling.

After having used the simplified model of a fully polarized $6 s$ shell and an empty $5 d$ one, for a more realistic description we take into account that both shells are partially occupied and polarized even for a $4 f^{n}$ RE adatom and, consequently, that the electrons in both shells are exchange coupled with the $4 f$ ones. Therefore, the spin-excitation energy corresponding to the reversal of the spin $S_{s d}$ is given by $\Delta E_{s d}=2 J_{4 f-s d} \Delta S_{s d} S_{4 f}$, where $J_{4 f-s d} \Delta S_{s d}=$ $J_{4 f-6 s} \Delta S_{6 s}+J_{4 f-5 d} \Delta S_{5 d}$. In order to evaluate $\Delta E_{s d}$, the intra-atomic exchange coupling constants, as well as the occupation and spin polarization of the outer shells for the adsorbed atoms, are required. To get these quantities, we carry out DFT calculations for a simplified configuration with the RE atoms adsorbed on freestanding graphene (for details, see Appendix B). Ignoring the effect of the $\mathrm{Cu}$ or Ir substrate is justified by the large graphene-metal substrate distance on both Ir [40] and $\mathrm{Cu}$ [41], being of the order of $3.3 \AA$. This distance results in a graphene electronic structure very similar to that of freestanding graphene $[42,43]$. The main effect is a minor charge transfer between the substrate and graphene, which is less than 0.01 electrons per $\mathrm{C}$ atom in the case of $\mathrm{Cu}$ [41]. All data suggest a weak van der Waals interaction between graphene and these substrates. The slightly different screening provided by the two metal substrates, inducing small differences in the charge transfer and polarization of the $6 s$ and $5 d$ shells, is likely at the origin of $E_{\text {ex }}$ values $10 \%$ lower on $\operatorname{gr} / \operatorname{Ir}(111)$ than on $\mathrm{gr} / \mathrm{Cu}$; see 
Fig. 1(c). The same arguments hold to explain the small variations of $E_{\text {ex }}$ observed between microcrystal grains on $\mathrm{gr} / \mathrm{Cu}$. However, these variations in screening are not expected to significantly change the intra-atomic exchange energies, and therefore, accurate calculations of these energies can be performed by neglecting the influence of the $\mathrm{Cu}$ or Ir substrate.

Within this refined description, we obtain the $\Delta E_{s d}$ values reported in Fig. 1(c) (black crosses) and in Table I (full model). The agreement between experimental and calculated values is substantially improved with respect to the one obtained with the simplified model. The main reason stems from the reduced spin polarization of the $S_{s d}$ shell calculated for the graphene-supported RE atoms. Nonetheless, we note that in the full model, the contribution of the $6 s$ shell to $\Delta E$ dominates over the $5 d$ one. This result reflects the IETS observations since a large $5 d$ spin polarization would result in much higher exchange energy, as $J_{4 f-5 d}$ is 2 to 3 times larger than $J_{4 f-6 s}$ (see Table I). The dominant $6 s$ character of the magnetic moment is in agreement with theoretical calculations of $\mathrm{Eu}$ on graphene [44]. The still-remaining deviation between theory and experiment reflects the complexity of these calculations and, in particular, the difficulty of calculating the correct bond distance between the RE atoms and graphene (see Appendix D). Our findings on the outer-shell polarization are in contrast with a recent theoretical work, which suggests a very small polarization of the $6 s$ and $5 d$ shells for Dy adatoms on $\operatorname{gr} / \operatorname{Ir}(111)$ [45]. The reason for this discrepancy is that the computational method used in Ref. [45] is a spin-polarized flavor of the Hubbard I approximation (see Ref. [46] for the details of the computational scheme). In this approach, the spin symmetry of the $4 f$ manifold is broken by an ad hoc field modeling the intra-atomic exchange, which leads to a magnetic solution. While this method is sufficient to describe the $4 f$ magnetism, the spin polarization of the $6 s$ and $5 d$ shells is underestimated since it is driven by the interatomic exchange, which is not taken into account in Ref. [45].

Further refinement of the description would include a better evaluation of the spin of the $4 f$ electrons of the RE adatoms. Multiplet calculations fitting x-ray absorption spectra for RE on graphene [25] show that $S_{4 f}$ is reduced by less than $5 \%$ with respect to the atomic values deduced from Hund's rules. To verify this analysis, we performed additional electronic structure calculations, where the $4 f$ electrons are treated in the Hubbard I approximation; see Appendix B. The largest reduction of $S_{4 f}$ is found for Dy and amounts to less than $3 \%$, accompanied by a change of the orbital moment $L_{4 f}$ of about $1 \%$. This result also confirms that the RE $4 f$ shell is only weakly perturbed by the adsorption on graphene.

Analysis of the IETS excitation linewidth $\delta E_{\text {ex }}$ gives access to the lifetime $\tau$ of the excited state (antiparallel configuration of $S_{s d}$ and $S_{4 f}$ ). Experimentally, $\delta E_{\mathrm{ex}}$ results from the intrinsic width $\hbar / \tau$, broadened by thermal effects and by the modulation voltage applied for the lock-in detection $[23,47,48]$. We find $\tau \approx 0.15 \mathrm{ps}$, independent of the RE element and of the supporting metal substrate. This value is slightly smaller than the one reported for the decay constant (0.8 ps) of the $5 d$ electrons in Gd [10]. In our system, the excitation originates mainly from the intraatomic exchange between $4 f$ and $6 s$ electrons. Given that the $6 s$ orbital is spatially more extended compared to the $5 d$, a stronger interaction with the support and thus a shorter lifetime is expected.

To further reinforce our interpretation of the IETS excitations, we investigate $\mathrm{Tb}$, known to have a $6 s^{1} 5 d^{1} 4 f^{n-1}$ type of configuration when adsorbed on graphene [25]. Indeed, our DFT calculations (not shown) predict that both $6 s$ and $5 d$ shells are partially occupied and spin polarized upon adsorption $\left(S_{6 s}=0.31, S_{5 d}=0.47\right)$. In the simplified description, this corresponds to $S_{6 s}=S_{5 d}=1 / 2$, spins that are strongly ferromagnetically coupled by a $J_{6 s-5 d}$ of about $0.5 \mathrm{eV}$ to form a spin for the outer shell $S_{s d}=1$ [19]. Only a partial breaking of the $s d-f$ exchange coupling can then be induced by a single spin-electron scattering event, corresponding to the transition of $S_{s d}$ from state \pm 1 to state 0 . This transition costs $\Delta E=2\left(J_{4 f-6 s}+J_{4 f-5 d}\right) \Delta S_{s d} S_{4 f} \approx 0.7 \mathrm{eV}$, which is estimated using $\Delta S_{s d}=1, S_{4 f}=3$, and published values of the Tb intra-atomic exchange constants $[18,19]$. Owing to instabilities in the junction induced for tunnel voltages exceeding $0.3 \mathrm{~V}$, we could not verify this excitation experimentally, signifying that, if that excitation exists, its energy is higher than $0.3 \mathrm{eV}$, as expected.

We note that other descriptions of the prominent IETS features displayed in Fig. 1 do not match our observations. Transitions between crystal-field split levels of the ground multiplet could have a linear dependence on $S_{4 f}$ in a uniaxial anisotropy scenario with similar anisotropy barriers for all RE species. However, previous works have shown that for each element, the energy-level scheme results from a richer set of anisotropy terms than the simple uniaxial one [24,25]. These higher-order terms have a strong impact on the level splitting, as clearly exemplified by the observation of high, low, or intermediate $m_{J 4 f}$ values for the adatom ground state, depending on the RE species [25]. Even in the realistic description of the crystal field including axial and transverse terms, transitions between levels can be excluded because (i) the observed excitation energies are 1 order of magnitude higher than the crystalfield splittings and (ii) no systematic dependence on $S_{4 f}$ is expected. Finally, excitations to the first excited multiplet can also be ruled out since the strong spin-orbit coupling, of the order of $200 \mathrm{meV}$, would lead to transitions at energies significantly higher than the observed ones $[4,33,39]$.

A vibrational origin can be excluded as well. In a simple description, with all the RE species adsorbed in equivalent graphene sites and with similar bond strength, the 
(a)

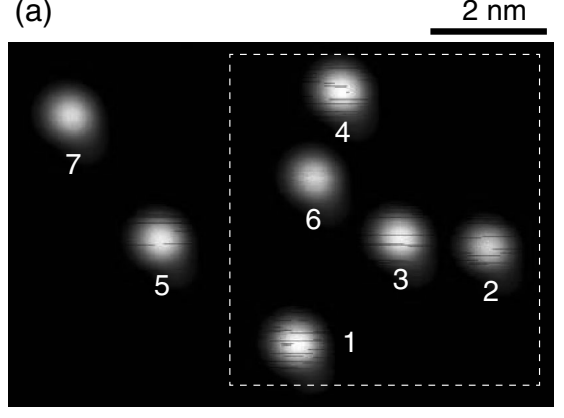

(b)

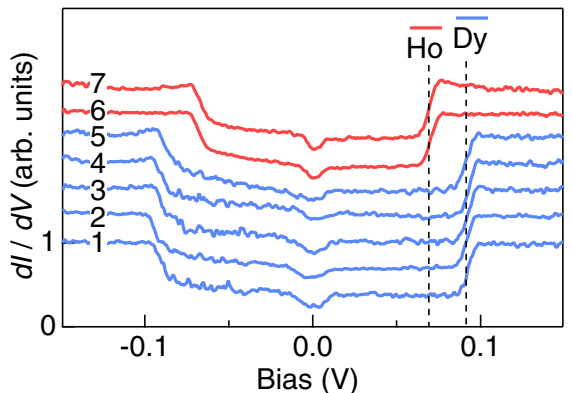

FIG. 3. Spin-polarized measurements on Dy and Ho on gr/Ir(111). (a) Constant current STM image showing the coexistence of Dy and Ho adatoms. The dashed white box identifies the region used for the video (see Ref. [53]). Parameters: $V_{\mathrm{t}}=+10 \mathrm{mV}, I_{\mathrm{t}}=20 \mathrm{pA}$, $T=6.5 \mathrm{~K}$. (b) $d I / d V$ spectra acquired on Dy and Ho adatoms. The spectra are background corrected, normalized to 1 , and vertically offset for clarity. Parameters: $I_{\text {set }}=100 \mathrm{pA}, V_{\text {set }}=-0.15 \mathrm{~V}, V_{\text {mod }}=5 \mathrm{mV}$ peak-to-peak, $T=6.5 \mathrm{~K}$. All data were acquired using a $\mathrm{Mn}_{88} \mathrm{Ni}_{12}$ tip.

vibrational energies scale with the inverse square root of the $\mathrm{RE}$ atomic masses [49]. The atomic masses increase only slightly $(20 \%)$ in going from $\mathrm{La}$ to $\mathrm{Yb}$; thus, the vibrational energies would follow a monotonic trend with a ratio of 1.1 between maximum (La) and minimum $(\mathrm{Yb})$ values. This behavior is clearly at variance with the trend and range of the experimental energies. Moreover, one would expect excitations for $\mathrm{La}, \mathrm{Yb}$, and $\mathrm{Tb}$, but we do not observe any. Finally, we also note that vibrational modes are expected at much lower energies. For example, for $\mathrm{Cs}$, an alkali species having both atomic mass and ionic bonding to graphene similar to the ones of the RE adatoms [31,50], vibrational modes were found at energies below $5 \mathrm{meV}$ [51].

To conclude this part, we note that a closer inspection of the spectra shown in Fig. 1(b) reveals the existence of other, less intense, inelastic features at lower bias. Their relatively low energy suggests a more involved origin than the intra-atomic exchange alone, probably related to transitions between the crystal-field split levels of the adatom.

The spin polarization of the RE adatom's outer shell can be assessed by observing the magnetoresistance in spinpolarized STM measurements. To this purpose, we performed experiments using out-of-plane spin-polarized tips made of $\mathrm{Mn}_{88} \mathrm{Ni}_{12}$, which is an antiferromagnetic alloy [52]. Figure 3(a) displays a constant current STM image acquired on a sample with coexisting Ho and Dy adatoms on $\operatorname{gr} / \operatorname{Ir}(111)$. The two species have similar apparent heights; however, the $d I / d V$ measurements shown in Fig. 3(b) allow us to unequivocally identify the two species by the characteristic energy of the inelastic features, as already shown in Fig. 1. A closer inspection of the STM image in Fig. 3(a) reveals that the Dy adatoms are unstable under the tunneling conditions used, with "broken" line scans testifying that they change apparent height several times during the scan. On the contrary, the Ho atoms appear with a constant height throughout the image.
The different dynamics of the two RE species is also evident in a movie that we generated from a series of 59 STM images acquired consecutively on the region indicated by the dashed box in Fig. 3(a), enclosing one Ho and four Dy atoms (see Ref. [53]). In those imaging conditions, the spin dynamics is slowed down with respect to Fig. 3(a), with the Dy atoms changing apparent height between successive frames and only rarely in a single frame, while the Ho atom keeps the same apparent height during the entire sequence of $1 \mathrm{~h} 4 \mathrm{~min}$. This observation is in agreement with previous measurements [25], revealing that on $\operatorname{gr} / \operatorname{Ir}(111)$, the magnetic relaxation time of Ho adatoms is at least 3 orders of magnitude shorter than the one of Dy. On the timescale of our observation, Ho atoms have a vanishing average projected magnetic moment as they frequently switch between the spin-up and spin-down states; consequently, they appear with a single average height, as for a paramagnetic atom.

The slow dynamics of Dy allows us to characterize the adatom polarization. Using a sequence of STM images acquired at tunnel parameters resulting in low switching frequencies, like the ones shown in Fig. 4(a), we determine the variation in apparent height for parallel vs antiparallel alignment of adatom and tip magnetic moments. Each adatom is labeled with $\mathrm{H}$ (high) or $\mathrm{L}$ (low) according to the height displayed when imaging its center (indicated by the red dots in the first frame). Analysis of the apparent height of the five atoms in eight successive frames yields the height distribution shown in Fig. 4(b). Two distinct values are found, separated by $\Delta z \approx 50 \mathrm{pm}$.

The two conductance states are caused by switching of the Dy magnetization. The histogram in Fig. 4(b) shows that the two states have nearly the same occupation. This observation indicates that spin torque [54] and tip stray or exchange field effects [52,55-57] are negligible at the tunneling conditions used and that magnetization reversal is mainly thermally induced. 


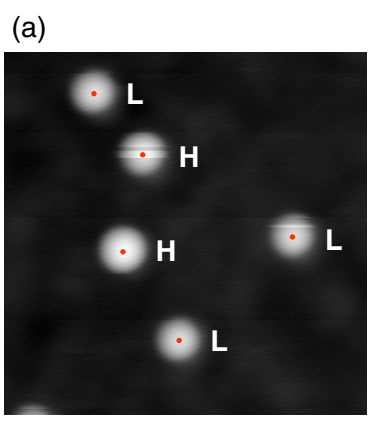

(b)

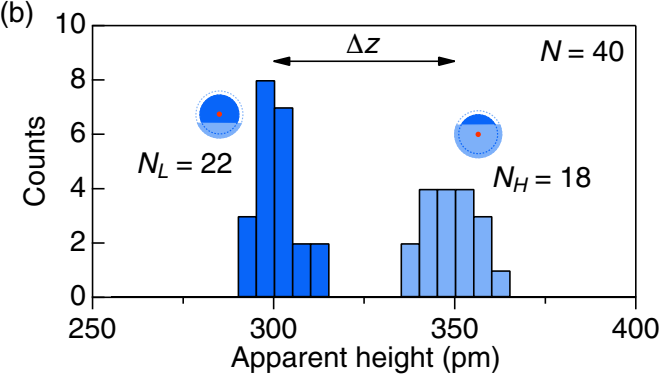

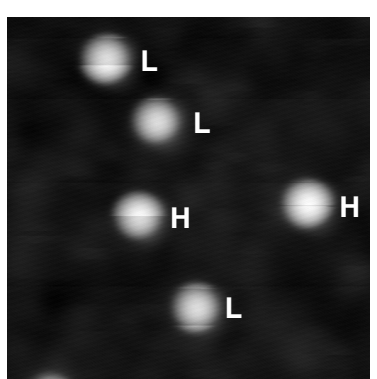
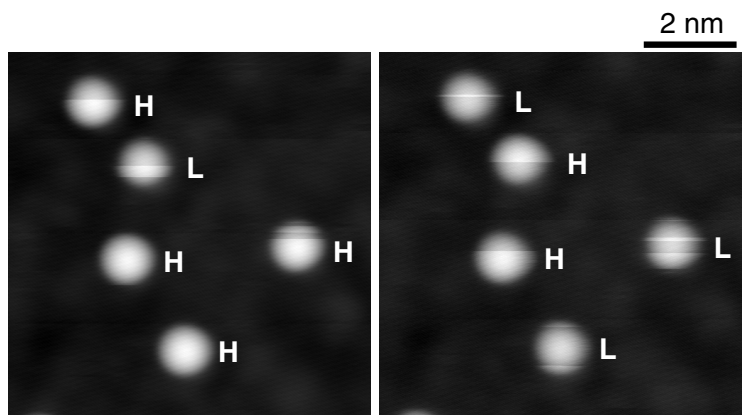

(c)

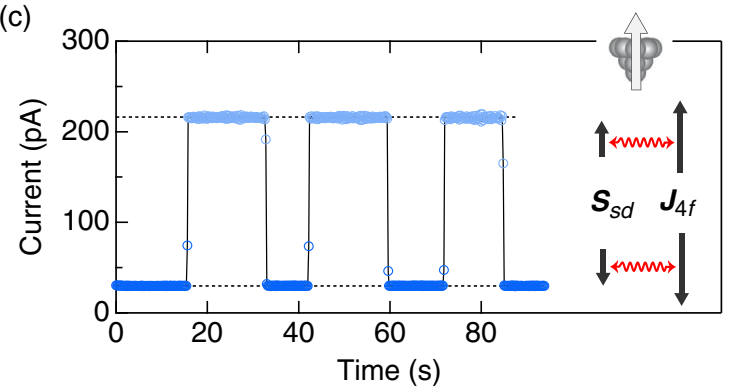

FIG. 4. Two-state telegraph signal in spin-polarized measurements for Dy/gr/Ir(111). (a) Sequence of constant current STM images showing Dy adatoms with switching apparent heights. $\mathrm{H}$ and L correspond to the height observed when scanning the protrusion center, indicated by a red dot in the first frame $\left(V_{\mathrm{t}}=+2 \mathrm{mV}, I_{\mathrm{t}}=20 \mathrm{pA}, T=6.5 \mathrm{~K}\right)$. (b) Histogram deduced from the apparent height of the five atoms in a sequence of eight images. (c) Constant height current trace acquired on a Dy adatom displaying magnetoresistive contrast due to the thermally excited switching of the Dy magnetization $\left(V_{\mathrm{t}}=+1 \mathrm{mV}, I_{\text {high }}=215 \mathrm{pA}, I_{\text {low }}=30 \mathrm{pA}, T=6.5 \mathrm{~K}\right)$. The sketch represents the Dy magnetization in the two degenerate states of the ground doublet with respect to the tip magnetization. All data were acquired using a $\mathrm{Mn}_{88} \mathrm{Ni}_{12}$ tip.

The magnetic ground state of the RE adatom is defined by $m_{J 4 f}$, the magnetic quantum number related to $\mathbf{J}_{4 f}$ resulting from the spin-orbit coupling of $\mathbf{S}_{4 f}$ with the orbital moment $\mathbf{L}_{4 f}$. Because $\mathbf{S}_{4 f}$ is coupled with $\mathbf{S}_{s d}$ via the intra-atomic exchange, $\mathbf{J}_{4 f}$ and $\mathbf{S}_{s d}$ flip simultaneously; see sketch on the right-hand side of Fig. 4(c). For the RE adatoms, tunneling takes place from/into the $6 s 5 d$ states since the $4 f$ electrons are highly localized and screened by the outer shells; moreover, the $4 f$ density of states (DOS) is zero at the Fermi energy [58]. Therefore, the observed contrast reflects the switching of $m_{S s d}$ caused by the thermal reversal of $m_{J 4 f}$ between the two states of the ground doublet defined by the crystal field [24,25].

The experimental $\Delta z \approx 50 \mathrm{pm}$ is very large with respect to the contrast previously observed on $3 d$ clusters [54,59] and on Ho adatoms [60], suggesting a high spin polarization of the Dy $6 s 5 d$ shells. In particular, the low contrast observed for $\mathrm{Ho} / \mathrm{MgO}$ can be ascribed to a weak polarization of the Ho outer shell, likely due to the different electronic configuration close to $6 s^{2} 5 d^{1} 4 f^{n-1}$ and to the nature of the bond with the oxygen.

To quantify the polarization of the Dy $6 s 5 d$ shell, we acquired constant-height current traces, showing a two-state telegraph signal. The junction polarization is determined from such current traces by the relation $P=$ $\left(I_{\text {high }}-I_{\text {low }}\right) /\left(I_{\text {high }}+I_{\text {low }}\right)$, where $I_{\text {high }}$ and $I_{\text {low }}$ are the high and low tunneling current values, respectively [61]. For $\mathrm{Dy} / \mathrm{gr} / \operatorname{Ir}(111)$, polarizations between $20 \%$ and $60 \%$ were routinely observed. In some cases, higher values were obtained, as for the data of Fig. 4(c), corresponding to $P=75 \%$. Usually, the spin polarization of STM junctions does not exceed $20 \%$, whether the measurements are on single atoms $[52,60,62,63]$, nanostructures $[54,59,64]$, or bulklike systems [61]; very high values have rarely been observed [65].

In a classical picture, the junction polarization can be described as $P \sim P_{\text {tip }} P_{\text {atom }} \cos \theta$, where $P_{\text {tip }}$ and $P_{\text {atom }}$ are tip and adatom spin polarization in the $E_{\mathrm{F}} \pm e V_{\text {bias }}$ range, respectively, and $\theta$ is the angle between the tip and adatom magnetic moments [66]. For our system, $P_{\text {atom }}$ corresponds to $P_{s d}$, the polarization of the outer shell, as tunneling takes place only from or into the $6 s 5 d$ states. Since only the electrons in the energy range $E_{F}-e V_{\text {bias }}$ contribute to the tunnel current and we use biases in the $\mathrm{mV}$ range, the observed magnetoresistance reflects the spin polarization very close to the Fermi energy.

A lower limit for $P_{s d}$ is obtained by assuming a parallel alignment of the tip and atom moment $(\theta=0)$ and a tip spin polarization $P_{\text {tip }}=100 \%$. The PDOS of $\mathrm{Mn}_{88} \mathrm{Ni}_{12}$ is unknown; however, $100 \%$ polarization at the Fermi energy has been calculated for similar Mn-based alloys $[67,68]$. With this assumption, we obtain $P_{s d}=75 \%$ at $E_{\mathrm{F}}$. This lower limit is fully compatible with the calculated 


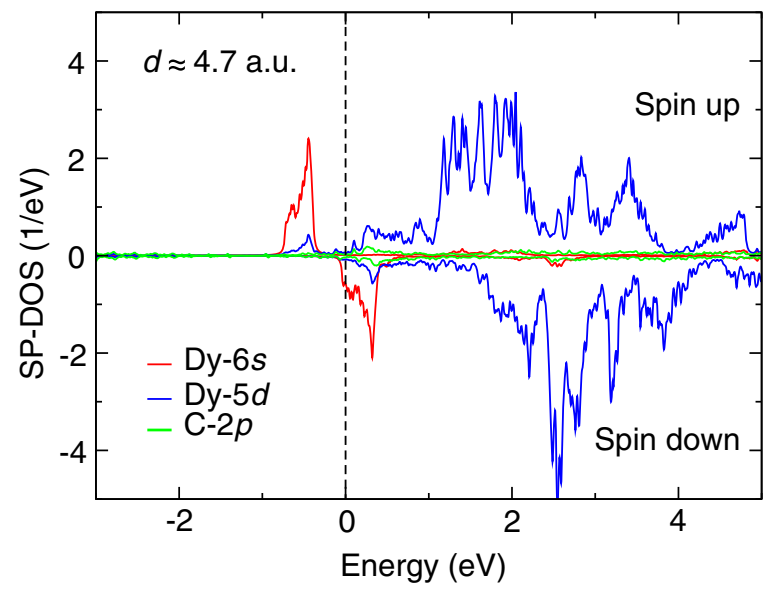

FIG. 5. Spin-polarized DOS around $E_{\mathrm{F}}$ for $6 s$ and $5 p$ shells of Dy and $2 p$ shell of $\mathrm{C}$. The dashed line marks $E_{\mathrm{F}}$. A smearing of 1 mRy has been applied.

spin-polarized DOS shown in Fig. 5 (see also Appendix D). In fact, we see that spin polarization at $E_{\mathrm{F}}$ is mainly determined by the $6 s$ states. The large exchange splitting leads to a predominance of the spin-down states, resulting in a calculated value for $P_{s d}$ of $100 \%$.

\section{CONCLUSIONS AND PERSPECTIVES}

Our paper reports the first measurement of the intraatomic exchange energies for several RE atoms. We show that a strong spin polarization can appear in the $6 s$ shell, leading to a dominant contribution of the $6 s$ shell to the intra-atomic $4 f-6 s 5 d$ coupling. Therefore, the frequently used description considering only the $4 f-5 d$ exchange is oversimplified, and interactions including all the external shells must be taken into account. The experimental values of these multishell interactions are of the order of tens of $\mathrm{meV}$. These energies are easily accessible, for instance, thermally or by optical means; therefore, the resulting excited states are expected to play a relevant role in the spin dynamics.

For example, in RE-based storage media, control of the ultrafast spin relaxation of each shell is necessary to realize fast reading and writing in new-generation magnetic memory devices. At the same time, single ions, adsorbed on a surface or enclosed in a molecular cage, are prototype systems for both classical and quantum bits. An improved rationalization of the spin-reversal mechanisms is a necessary requirement to open the path toward high-temperature single-ion magnets and quantum applications. In this context, only recently, the intra-atomic exchange interaction has been proposed to be a key parameter in the description of the magnetic level scheme and thus of the spin relaxation paths [69-71]. The coupling via the intraatomic exchange between the spins of $4 f$ and $6 s 5 d$ shells has an effect analogous to the one of hyperfine coupling between electron and nuclear spins but on a different energy scale, owing to the different strength of the exchange interaction. In this description, the quantum tunneling of magnetization (QTM) is not determined by $\mathbf{J}_{4 f}$ alone but by the total moment $\mathbf{J}_{4 f}+\mathbf{S}_{s d}$. For example, the magnetic hysteresis and remanence observed in Ho adatoms on $\mathrm{MgO}$ have been attributed to the missing QTM between the states of the ground doublet with $m_{J 4 f}= \pm 7$ in a crystal field with $C_{4 v}$ symmetry [72]. However, the $\mathrm{Ho} / \mathrm{MgO}$ magnetic behavior can also be explained by the missing QTM of a Kramer ground doublet, resulting in the exchange coupled $m_{J 4 f}= \pm 8$ and $m_{S s d}$, the latter moment originating from the partially polarized $5 d^{1}$ shell.

In addition, our findings demonstrate that spin dynamics can also involve excited states originating from the intraatomic exchange and precisely quantify the energy required for these processes.

\section{ACKNOWLEDGMENTS}

We acknowledge E. Fernandes for his contribution to the early stage of this work. We acknowledge valuable discussions with S. Panda. I. D. M. acknowledges support from the JRG Program at APCTP through the Science and Technology Promotion Fund and Lottery Fund of the Korean Government, as well as from the Korean Local Governments-Gyeongsangbuk-do Province and Pohang City. The computations were performed with resources provided by the Swedish National Infrastructure for Computing (SNIC) at the National Supercomputer Center (NSC). O.E. acknowledges support from the Swedish Research Council (VR), the Swedish Foundation for Strategic Research (SSF), the Knut and Alice Wallenberg Foundation, and eSSENCE.

\section{APPENDIX A: EXPERIMENTAL METHODS}

We used both commercial graphene (gr) on polycrystalline $\mathrm{Cu}$ (6Carbon Technology) and in situ CVD-grown graphene on $\operatorname{Ir}(111)$. The $\mathrm{gr} / \mathrm{Cu}$ was annealed in vacuum to $800 \mathrm{~K}$ to remove adsorbates after exposure to air. The $\operatorname{Ir}(111)$ single crystal was prepared by $\mathrm{Ar}^{+}$sputtering and annealing cycles $[24,42]$. Graphene was grown in situ by chemical vapor deposition from ethylene $(100 \mathrm{~L}$ at $1400 \mathrm{~K})$. RE atoms were deposited from thoroughly degassed high-purity rods (99.9\%) using an $e$-beam evaporator with a typical flux of $2 \times 10^{-3}$ monolayers (ML) per min, in a background pressure of $1 \times 10^{-10}$ mbar. The substrate temperature during deposition was approximately $10 \mathrm{~K}$, a temperature at which surface diffusion is inhibited, resulting in a random spatial distribution of adatoms [29]. The STM images were acquired in constant-current mode with the tunnel voltage $V$ applied to the sample [73]. The differential conductance $d I / d V$ spectra were acquired using lock-in detection, with modulation $V_{\text {mod }}=$ 1-5 $\mathrm{mV}$ peak-to-peak at $397 \mathrm{~Hz}$ added to the bias voltage $V$. Chemically etched $\mathrm{W}$ and $\mathrm{Mn}_{88} \mathrm{Ni}_{12}$ [52] tips were used. 


\section{APPENDIX B: THEORETICAL METHODS}

Electronic structure calculations were performed using the DFT code RSPt [74]. RSPt is based on the full-potential linear muffin-tin orbital (FP-LMTO) method and belongs to the most accurate family of all-electron DFT codes [75]. The exchange-correlation potential was treated in the generalized gradient approximation (GGA), using the parametrization by Perdew, Burke, and Enzerhof [76]. The valence electrons of $\mathrm{C}$ were described using $2 s, 2 p$, and $3 d$ derived states. The valence electrons of the RE atoms were described using $6 s, 6 p, 5 d$, and $5 f$ states in a first energy set, and $5 s$ and $5 p$ states in a second energy set. The localized $4 f$ states were treated as open-core states, using the fully relativistic Dirac equation $[26,77,78]$. The size of the muffin-tin spheres, which in a full-potential code are used to divide the physical space in the unit cell, was optimized to minimize the leakage of the core states. This procedure led to muffin-tin radii of 1.27 a.u. for $\mathrm{C}$ and 3.02 a.u. for RE atoms. A proper description of the wave functions in the interstitial region required the usage of four kinetic energy tails $\kappa^{2}$ for the late rare-earth atoms and of five kinetic energy tails $\kappa^{2}$ for the early rare-earth atoms.

The experimental systems were modeled by considering a single RE adatom on freestanding graphene. Supercells were constructed with a $3 \times 3$ graphene sheet with a RE atom in the hollow position, in analogy to Ref. [79] and in agreement with experiments [24], and a vacuum region of at least $17 \AA$. The Brillouin zone was sampled with a dense Monkhorst-Pack grid of $24 \times 24 \times 1$ k-points. With this computational setup, full structural relaxations were performed for both trivalent and divalent RE adatoms. The analysis of the total energies as in Ref. [77] showed that $\mathrm{Sm}, \mathrm{Eu}, \mathrm{Dy}, \mathrm{Ho}, \mathrm{Er}, \mathrm{Tm}$, and $\mathrm{Yb}$ prefer a $4 f^{n}$ configuration, while $\mathrm{La}$ and $\mathrm{Tb}$ resulted in $4 f^{n-1}$. The distance between the graphene plane and the adatoms in their $4 f^{n}$ configuration is around 4.70 a.u., with small variations depending on the atomic number. These values are in good agreement with the value of 4.58 a.u obtained in Ref. [79] for a Sm atom on graphene.

The relaxed structures were then used to perform magnetic, fully relativistic calculations, where the $4 f$ states were treated as a spin-polarized core [4]. In practice, this means that spin and orbital moments of the $4 f$ shell follow Russel-Sunders coupling and are in accordance with Hund's rules, through the occupation of the solutions of the Koelling-Harmon equations [80]. Because of the large extension of the RE $6 s$ states, locally projected quantities were obtained by directly using the native LMTOs and not projectors limited to the muffin-tin spheres, as is usually done in augmented methods [74]. To ensure the convergence of all quantities, finer $\mathbf{k}$-meshes were tested up to $81 \times 81 \times 1$.

To analyze the quenching of $\mathbf{S}$ due to the crystal field, we also performed DFT + DMFT calculations [81] in the Hubbard I approximation [82,83], still using RSPt. The computational parameters were defined as above but replacing the $5 f$ states in the basis with $4 f$ states, which were removed from the core. Calculations were performed at room temperature in the paramagnetic phase, using the same Coulomb interaction parameters previously used for the RE metals [83]. The double counting was treated as in the fully localized limit with nominal atomic occupations, as routinely used for RE systems [83-85]. Overall, this approach provides a good description of the multiplet spectrum of the $4 f$ shell and allows for the analysis of the competition between Coulomb interaction and crystal field, at least in the paramagnetic phase. In the magnetically ordered phase, instead, this approach suffers from a loss of precision, due to the unknown double-counting term, as amply discussed in Refs. $[45,46,86]$. Thus, we chose to perform the investigation of the magnetic properties through the open-core treatment of the $4 f$ states.

The calculations of the intra-atomic exchange were performed [87] using atomiclike wave functions, which coincide with the muffin-tin heads of the LMTOs [74]. The extended part of the wave functions, which is important to describe the magnetic moments (see discussion above), has no relevance for the values of the exchange integrals since our tests show that they decay much faster. Note that in the formalism of Ref. [87], only the isotropic contribution to the exchange is considered since the anisotropic terms were shown to be negligible in the lanthanides [18]. Screening was considered in a Yukawa form [88], where the screening length was determined to ensure that the strength of the average local Coulomb interaction of $4 f$ electrons coincides with values calculated via constrained density functional theory.

\section{APPENDIX C: DISTINGUISHING CLEAN FROM CONTAMINATED RE ATOMS}

Investigation of the intrinsic properties of clean, individual $\mathrm{RE}$ adatoms on a surface requires the cleanest possible conditions. A first essential step is a careful, thorough degassing of the evaporation materials. For the high-purity RE rods, clean depositions typically necessitate day-long degassing cycles, in order to reach a background pressure in the low $10^{-10}$ mbar during deposition.

In the following, we describe experiments that unambiguously distinguished clean from contaminated RE adatoms. Figure 6(a) shows a typical STM image acquired on a Dy on a gr/Ir(111) sample prepared after careful Dy degassing, Dy deposition at $10 \mathrm{~K}$, and a Dy coverage of $0.5 \%$ ML. Most of the protrusions (88\% of the objects) have an apparent height between 450 and $500 \mathrm{pm}$ at the tunneling parameters used (orange arrow). They are identified as clean Dy adatoms [24,29]. Owing to a large direct impingement area, many dimers are formed during deposition [29]. They appear as lower (350 to $400 \mathrm{pm}$ ), slightly elongated objects (yellow arrow) and can be split by applying a tunnel voltage ramp [24]. For this coverage, 

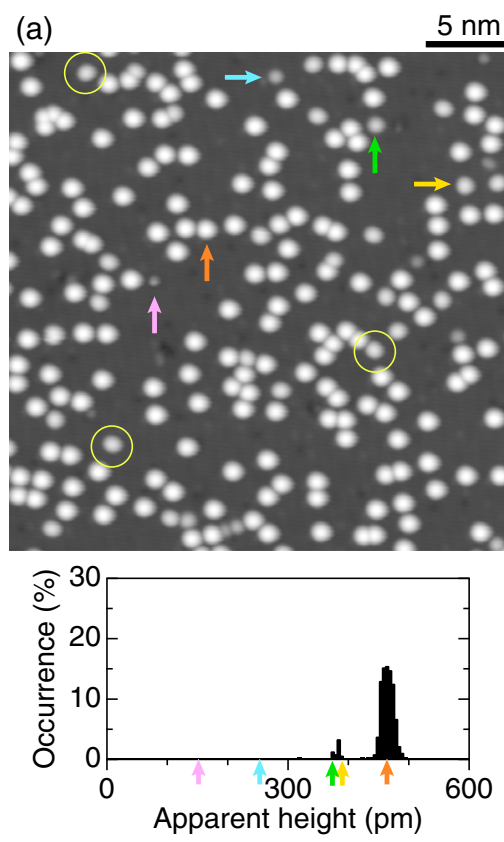

(b)
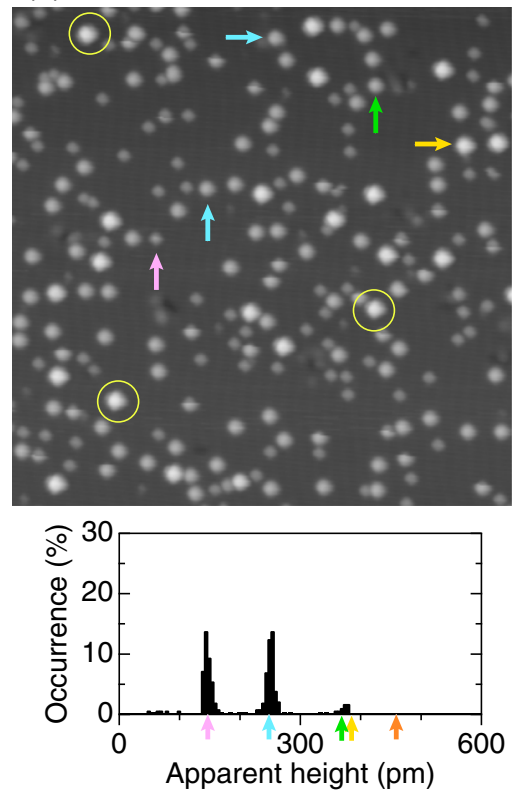

Exposure to residual gas (c)
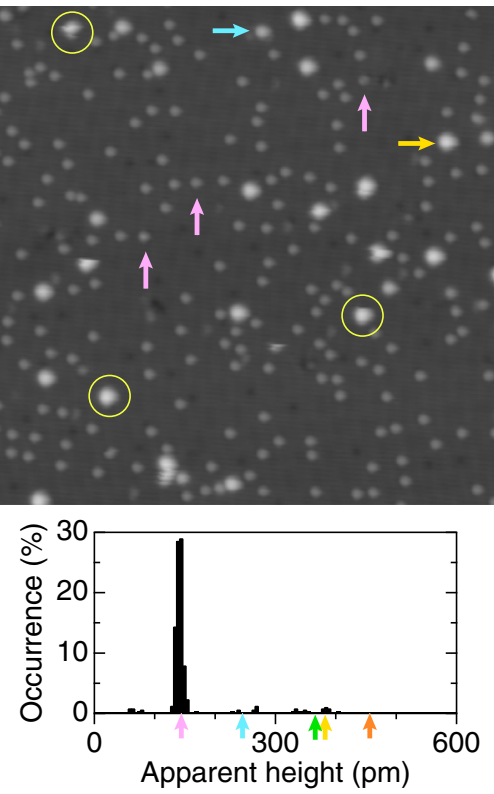

FIG. 6. Distinguishing clean and hydrogenated RE adatoms. Sequence of STM images of Dy on gr/Ir(111) acquired before, during, and after exposure to gas, mainly $\mathrm{H}_{2}$, desorbing from the cryostat walls upon heating up the STM. (a) Reference STM image: The arrows indicate a clean Dy atom (orange), a Dy dimer (yellow), and contaminated atoms (green, cyan, and pink). Yellow circles highlight some Dy dimers that are used as landmarks $\left(T=6.5 \mathrm{~K}, p=1 \times 10^{-10} \mathrm{mbar}\right)$. (b) Same region during $\left(T=20 \mathrm{~K}, p=3 \times 10^{-9} \mathrm{mbar}\right)$ and (c) after $\left(T=33 \mathrm{~K}, p=3 \times 10^{-10}\right.$ mbar) massive exposure to $\mathrm{H}_{2}$ desorbing from the cryogenic shields. The arrows indicate the same objects as in panel (a), with the colors adapted according to the new apparent heights. Parameters for all images: $V_{\mathrm{t}}=-0.5 \mathrm{~V}$, $I_{\mathrm{t}}=20 \mathrm{pA}, \Theta_{\mathrm{Dy}}=0.5 \% \mathrm{ML}, \mathrm{W}$ tip. The bottom panels show histograms of the observed apparent heights for each measurement, with statistics on approximately 450 protrusions each.

they represent $7 \%$ of the objects. In addition to clean monomers and dimers, there are very few protrusions with apparent height similar to the one of dimers, but with round shape (green arrow), as well as some protrusions displaying even lower apparent heights (cyan and pink arrows). The percentage of monomers vs dimers depends on the coverage: For a coverage of $0.1 \% \mathrm{ML}$ or less, exclusively monomers will be found [29]. The histogram in the bottom panel shows the occurrence of the different species (statistics on approximately 450 objects). Overall, this sample presents $95 \%$ clean Dy species and 5\% of the species with $\mathrm{H}$ adsorbates, as discussed below.

It is also essential to prevent contamination once the atoms are on the surface. In our setup, the pressure inside the LHe-cooled radiation shields, where the sample is located, is estimated to be below $10^{-14} \mathrm{mbar}$. In these conditions, no measurable contamination has been observed $[73,89]$. However, to investigate the effect of the residual gas on the RE adatoms, we have carried out the following experiment: After having prepared the sample with the standard procedure and having characterized it at $6.5 \mathrm{~K}$, we let the cryostat heat while continuing the measurements. At around $16 \mathrm{~K}$, the background pressure starts to rise because of residual gas desorbing from the cryostat and the shield walls. At this temperature, desorption of $\mathrm{H}_{2}$ is expected [89].

The sample evolution was monitored by imaging the same spot during the temperature and pressure change. The STM images in Figs. 6(b) and (c) were acquired, respectively, during, at $T=20 \mathrm{~K}$ and $p=3 \times 10^{-9} \mathrm{mbar}$, and after the pressure peak, at $T=33 \mathrm{~K}$ and $p=3 \times 10^{-10} \mathrm{mbar}$. In Fig. 6(b), most of the objects have changed apparent height. However, dimers did not change their appearance and can be used as landmarks: Some of them are encircled in yellow. All the Dy adatoms show up with much lower height. Also, the object indicated by the green arrow now has a lower height. Two families of small objects can be identified, with height of approximately $250 \mathrm{pm}$ and $150 \mathrm{pm}$, corresponding to the heights of the protrusions marked by the cyan and pink arrows, respectively, which is clearly visible on the corresponding histogram in the bottom panel. With time, since the residual $\mathrm{H}_{2}$ is still present at the surface of the sample even after the pressure peak, almost all the cyan protrusions transform into pink ones, as demonstrated in the STM image and the histogram in Fig. 6(c). A reduction of the apparent height upon single and multiple $\mathrm{H}$ adsorption has been reported for transition metal atoms adsorbed on similar surfaces $[89,90]$. 
(a)

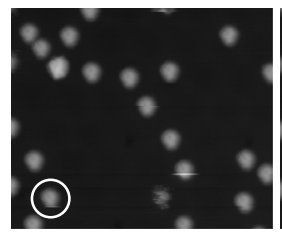

(b)

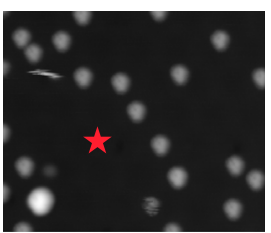

(c)

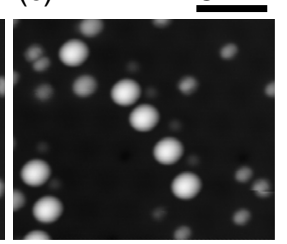

FIG. 7. Manipulation procedures for $\mathrm{H}$ removal from Dy adatoms. We show a sequence of STM images acquired on a Dy on $\mathrm{gr} / \operatorname{Ir}(111)$ sample that was exposed to gas desorbing from the cryostat walls upon heating up the STM. (a) Reference STM image: A voltage ramp ( -0.5 to $-2 \mathrm{~V}$, closed feedback loop) is then applied on the encircled hydrogenated atom to clean it, as is visible in panel (b). (b) A-4 V voltage pulse (50 ms, open feedback loop) applied on the graphene substrate at the location indicated by the red star. (c) After the voltage pulse, several clean Dy adatoms are present. Parameters for all images: $V_{\mathrm{t}}=-0.5 \mathrm{~V}$, $I_{\mathrm{t}}=20 \mathrm{pA}, T=6.5 \mathrm{~K}, \Theta_{\mathrm{Dy}}=0.5 \% \mathrm{ML}, \mathrm{W}$ tip.

The contaminated Dy adatom can be cleaned by manipulation, and various procedures have been tested [89]. Figure 7(a) shows a STM image acquired on Dy on gr/Ir(111) after exposure to residual gas, with the same procedure described for Fig. 6. Applying a voltage ramp ( -0.5 to $-2 \mathrm{~V}$, closed feedback loop) on the hydrogenated atom encircled in white results in a clean Dy monomer, as visible in Fig. 7(b). Subsequently, a -4 V pulse ( $50 \mathrm{~ms}$, open feedback loop) applied at the location indicated by the red star removes the hydrogen from the nearby Dy atoms, as shown in the image in Fig. 7(c), on which seven clean Dy monomers are visible. Note that on the image shown in Fig. 7, the Dy adatoms show some ordering with respect to the moiré pattern periodicity because of the sample preparation conditions [24,29].
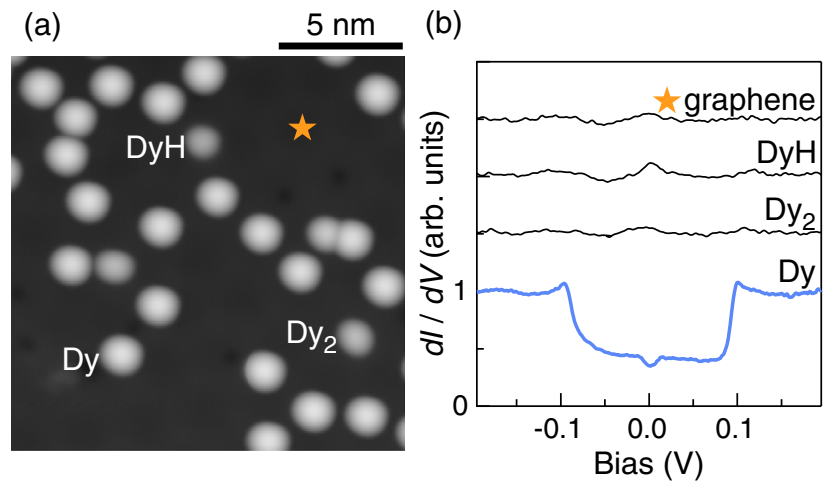

FIG. 8. The $d I / d V$ spectroscopic signatures. (a) STM image acquired on Dy on gr/Ir(111). Labels identify a Dy monomer, a Dy dimer, and a hydrogenated Dy. Parameters: $V_{\mathrm{t}}=-0.2 \mathrm{~V}$, $I_{\mathrm{t}}=100 \mathrm{pA}, T=6.5 \mathrm{~K}, \Theta_{\text {Dy }}=0.5 \% \mathrm{ML}$. (b) $d I / d V$ spectra acquired on a Dy atom, a Dy dimer, a hydrogenated Dy atom, and on the graphene substrate. All spectra are background corrected and normalized to 1 and vertically offset for clarity. Parameters: $I_{\text {set }}=150 \mathrm{pA}, \quad V_{\text {set }}=-0.2 \mathrm{~V}, \quad V_{\text {mod }}=5 \mathrm{mV}$ peak-to-peak, $T=6.5 \mathrm{~K}$. All data were acquired using a $\mathrm{W}$ tip.
Finally, we have also characterized the contaminated Dy atoms by their differential conductance spectra. Figure 8(a) shows a STM image on which many Dy adatoms, two Dy dimers (slightly elongated shape), and a hydrogenated Dy atom are visible. Some of the objects are identified by labels. Figure 8(b) shows $d I / d V$ spectra acquired on a Dy atom, a Dy dimer, a hydrogenated Dy atom, and on the graphene substrate. Only the spectrum measured on the clean Dy atom displays the characteristic inelastic steps, like the ones shown in Fig. 1, which gives an additional tool to distinguish between clean Dy adatoms and other species. The absence of the steps due to intra-atomic exchange coupling on the hydrogenated species can be rationalized by hydrogen adsorption, implying bonding to the $s d$ shell, resulting in a loss of the shell spin polarization. Similarly, the occupation and/or polarization of the outer shell is modified for Dy dimers, resulting in the absence of inelastic features related to the intra-atomic exchange.

\section{APPENDIX D: ROLE OF ADATOM-GRAPHENE DISTANCE IN DFT}

The exchange integrals and the overall spin polarization of the $6 s$ and $5 d$ shells of the RE adatom are influenced by

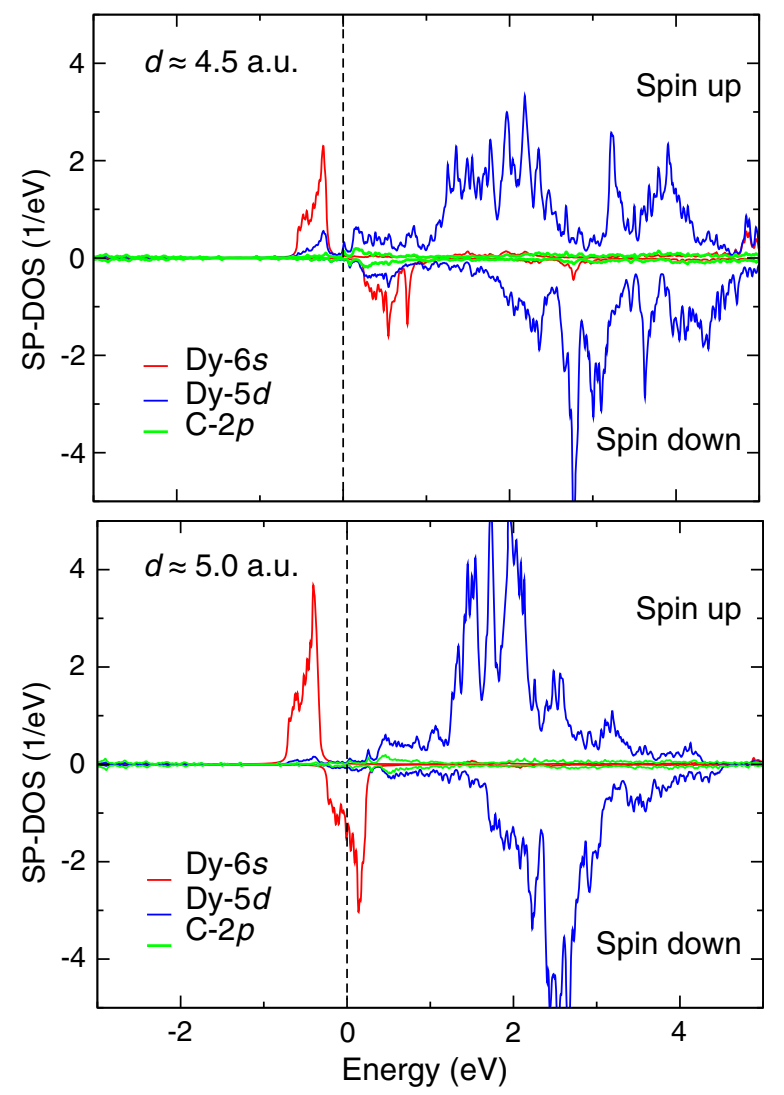

FIG. 9. Spin-polarized DOS around $E_{\mathrm{F}}$ for $6 s$ and $5 p$ shells of $\mathrm{Dy}$, and a $2 p$ shell of $\mathrm{C}$, for two Dy-graphene adsorption distances (4.5 a.u. and 5.0 a.u.). The dashed line marks $E_{\mathrm{F}}$. A smearing of $1 \mathrm{mRy}$ has been applied. 
the distance $d$ between the adatom and the graphene layer. For instance, calculations similar to the ones presented in Fig. 1(c) and Table I, but with a bond distance reduced by $3 \%$, reduce the intra-atomic exchange energy by $5 \%-10 \%$ depending on the element (data not shown). Similarly, the exchange splitting and the position of the Fermi level were found to be rather sensitive to the bond distance, which is, in turn, dependent on the DFT functional and on the computational details used for the calculations (compare, e.g., Refs. [30,79]). This effect is illustrated in Fig. 9, where the SP-DOS of Dy on graphene is reported for equilibrium distances $d$ obtained with different computational settings. Experience shows that theories based on GGA have toolarge bond distances (with 1\%) for light rare earths, while for late rare earths, the bond distance is reproduced with almost perfect agreement. This aspect is discussed in detail in Ref. [77].

The reason for the relatively strong influence of the $6 s$ projected electronic structure with respect to bond distance is the different extent of the $6 s$ and $5 d$ wave functions. The formation of hybridized states involving $5 d$ orbitals changes significantly when moving from large bond distances to short ones. This change is particularly evident in the dependence of the number of $5 d$ states hybridizing with the $6 s$ majority spin band with respect to the bond length.

Finally, we note that the SP-DOS shown in Figs. 5 and 9 is limited to the region around the Fermi energy, which gives the largest contribution to the local magnetic moments. States with $6 s$ and $5 d$ character also appear at lower energies, but they contribute mainly to the local charge.

[1] Rare Earth Doped III-Nitrides for Optoelectronic and Spintronic Applications, edited by K. O'Donnell and V. Dierolf (Springer, New York, 2010).

[2] M. Rancic, M. P. Hedges, R. L. Ahlefeldt, and M. J. Sellars, Coherence Time of Over a Second in a Telecom-Compatible Quantum Memory Storage Material, Nat. Phys. 14, 50 (2018).

[3] D. Serrano, J. Karlsson, A. Fossati, A. Ferrier, and P. Goldner, All-Optical Control of Long-Lived Nuclear Spins in Rare-Earth Doped Nanoparticles, Nat. Commun. 9, 2127 (2018).

[4] J. Jensen and A. R. Mackintosh, Rare Earth Magnetism, Structures and Excitations (Clarendon Press, Oxford, 1991).

[5] J. M. D. Coey, ed., Rare-Earth Iron Permanent Magnets, edited by J. M. D. Coey (Clarendon Press, Oxford, 1996).

[6] J. F. Herbst, $\mathrm{R}_{2} \mathrm{Fe}_{14} \mathrm{~B}$ Materials: Intrinsic Properties and Technological Aspects, Rev. Mod. Phys. 63, 819 (1991).

[7] A. Melnikov, I. Radu, A. Povolotskiy, T. Wehling, A. Lichtenstein, and U. Bovensiepen, Ultrafast Dynamics at Lanthanide Surfaces: Microscopic Interaction of the Charge, Lattice and Spin Subsystems, J. Phys. D 41, 164004 (2008).
[8] B. B. Koopmans, G. Malinowski, F. Dalla Longa, D. Steiauf, M. Fähnle, T. Roth, M. Cinchetti, and M. Aeschlimann, Explaining the Paradoxical Diversity of Ultrafast Laser-Induced Demagnetization, Nat. Mater. 9, 259 (2010).

[9] M. Wietstruk, A. Melnikov, C. Stamm, T. Kachel, N. Pontius, M. Sultan, C. Gahl, M. Weinelt, H. A. Dürr, and U. Bovensiepen, Hot-Electron-Driven Enhancement of Spin-Lattice Coupling in $G d$ and $T b 4 f$ Ferromagnets Observed by Femtosecond X-Ray Magnetic Circular Dichroism, Phys. Rev. Lett. 106, 127401 (2011).

[10] B. Frietsch, J. Bowlan, R. Carley, M. Teichmann, S. Wienholdt, D. Hinzke, U. Nowak, K. Carva, P. M. Oppeneer, and M. Weinelt, Disparate Ultrafast Dynamics of Itinerant and Localized Magnetic Moments in Gadolinium Metal, Nat. Commun. 6, 8262 (2015).

[11] L. Rettig, C. Dornes, N. Thielemann-Kühn, N. Pontius, H. Zabel, D. L. Schlagel, T. A. Lograsso, M. Chollet, A. Robert, M. Sikorski, S. Song, J. M. Glownia, C. SchüßlerLangeheine, S. L. Johnson, and U. Staub, Itinerant and Localized Magnetization Dynamics in Antiferromagnetic Ho, Phys. Rev. Lett. 116, 257202 (2016).

[12] G. P. Zhang, T. Jenkins, M. Bennett, and Y.H. Bai, Manifestation of Intra-atomic 5d6s-4f Exchange Coupling in Photoexcited Gadolinium, J. Phys. Condens. Matter 29, 495807 (2017).

[13] R. R. Subkhangulov, A. B. Henriques, P. H. O. Rappl, E. Abramof, T. Rasing, and A. V. Kimel, All-Optical Manipulation and Probing of the $d-f$ Exchange Interaction in EuTe, Sci. Rep. 4, 4368 (2015).

[14] N. Bergeard, V. López-Flores, V. Halté, M. Hehn, C. Stamm, N. Pontius, E. Beaurepaire, and C. Boeglin, Ultrafast Angular Momentum Transfer in Multisublattice Ferrimagnets, Nat. Commun. 5, 3466 (2014).

[15] M. Matsubara, A. Schroer, A. Schmehl, A. Melville, C. Becher, M. Trujillo-Martinez, D. G. Schlom, J. Mannhart, J. Kroha, and M. Fiebig, Ultrafast Optical Tuning of Ferromagnetism via the Carrier Density, Nat. Commun. 6, 6724 (2015).

[16] S. H. Liu, Exchange Interaction between Conduction Electrons and Magnetic Shell Electrons in Rare-Earth Metals, Phys. Rev. 121, 451 (1961).

[17] F. Milstein and L. B. Robinson, Exchange Integral in RareEarth Metals, Phys. Rev. Lett. 18, 308 (1967).

[18] H.-S. Li, Y. P. Li, and J. M. D. Coey, $R-T$ and $R-R$ Exchange Interactions in the Rare-Earth (R)-TransitionMetal (T) Intermetallics: An Evaluation from Relativistic Atomic Calculations, J. Phys. Condens. Matter 3, 7277 (1991).

[19] R. Ahuja, S. Auluck, B. Johansson, and M. S. S. Brooks, Electronic Structure, Magnetism, and Fermi Surfaces of Gd and Tb, Phys. Rev. B 50, 5147 (1994).

[20] J. A. Heinrich, A. J. Gupta, C. P. Lutz, and D. M. Eigler, Single-Atom Spin-Flip Spectroscopy, Science 306, 466 (2004).

[21] C. F. Hirjibehedin, C.-Y. Lin, A. F. Otte, M. Ternes, C. P. Lutz, B. A. Jones, and A. J. Heinrich, Large Magnetic Anisotropy of a Single Atomic Spin Embedded in a Surface Molecular Network, Science 317, 1199 (2007). 
[22] A. F. Otte, M. Ternes, K. von Bergmann, S. Loth, H. Brune, C. P. Lutz, C. F. Hirjibehedin, and A. J. Heinrich, The Role of Magnetic Anisotropy in the Kondo Effect, Nat. Phys. 4, 847 (2008).

[23] A. A. Khajetoorians, S. Lounis, B. Chilian, A. T. Costa, L. Zhou, D. L. Mills, J. Wiebe, and R. Wiesendanger, Itinerant Nature of Atom-Magnetization Excitation by Tunneling Electrons, Phys. Rev. Lett. 106, 037205 (2011).

[24] R. Baltic, M. Pivetta, F. Donati, C. Wäckerlin, A. Singha, J. Dreiser, S. Rusponi, and H. Brune, Superlattice of Single Atom Magnets on Graphene, Nano Lett. 16, 7610 (2016).

[25] R. Baltic, F. Donati, A. Singha, C. Wäckerlin, J. Dreiser, B. Delley, M. Pivetta, S. Rusponi, and H. Brune, Magnetic Properties of Single Rare-Earth Atoms on Graphene/ Ir(111), Phys. Rev. B 98, 024412 (2018).

[26] A. Delin, L. Fast, B. Johansson, J. M. Wills, and O. Eriksson, Method for Calculating Valence Stability in Lanthanide Systems, Phys. Rev. Lett. 79, 4637 (1997).

[27] A. Singha, R. Baltic, F. Donati, C. Wäckerlin, J. Dreiser, L. Persichetti, S. Stepanow, P. Gambardella, S. Rusponi, and H. Brune, $4 \mathrm{f}$ Occupancy and Magnetism of Rare-Earth Atoms Adsorbed on Metal Substrates, Phys. Rev. B 96, 224418 (2017).

[28] F. Donati, A. Singha, S. Stepanow, C. Wäckerlin, J. Dreiser, P. Gambardella, S. Rusponi, and H. Brune, Magnetism of Ho and Er Atoms on Close-Packed Metal Surfaces, Phys. Rev. Lett. 113, 237201 (2014).

[29] M. Pivetta, S. Rusponi, and H. Brune, Direct Capture and Electrostatic Repulsion in the Self-Assembly of Rare-Earth Atom Superlattices on Graphene, Phys. Rev. B 98, 115417 (2018).

[30] X. Liu, C. Z. Wang, M. Hupalo, W. C. Lu, M. C. Tringides, Y. X. Yao, and K. M. Ho, Metals on Graphene: Correlation between Adatom Adsorption Behavior and Growth Morphology, Phys. Chem. Chem. Phys. 14, 9157 (2012).

[31] D. F. Förster, T. O. Wehling, S. Schumacher, A. Rosch, and T. Michely, Phase Coexistence of Clusters and Islands: Europium on Graphene, New J. Phys. 14, 023022 (2012).

[32] Magnetism in Condensed Matter, edited by S. Blundell (Oxford University Press, New York, 2001).

[33] J. Stör and H. C. Siegmann, Magnetism-From Fundamentals to Nanoscale Dynamics (Springer, Berlin, 2006).

[34] L. E. Roy and T. Hughbanks, Magnetic Coupling in Dinuclear Gd Complexes, J. Am. Chem. Soc. 128, 568 (2006).

[35] P. M. Levy, The Evidence for Anisotropic Rare-EarthConduction Electron Interactions, J. Phys. Colloques 40, C5 (1979).

[36] P.-G. de Gennes, Sur les Propriétés des Métaux des Terres Rares, C.R. Acad. Sci. 247, 1836 (1958).

[37] P.-G. de Gennes, Interactions Indirectes Entre Couches $4 f$ Dans les Métaux de Terres Rares, J. Phys. Radium 23, 510 (1962).

[38] L. L. Hirst, Theory of the Coupling between Conduction Electrons and Moments of $3 d$ and $4 f$ Ions in Metals, Adv. Phys. 27, 231 (1978).

[39] R. Skomski, Simple Models of Magnetism (Oxford University Press, New York, 1996).
[40] C. Busse, P. Lazić, R. Djemour, J. Coraux, T. Gerber, N. Atodiresei, V. Caciuc, R. Brako, A. T. N'Diaye, S. Blügel, J. Zegenhagen, and T. Michely, Graphene on $\operatorname{Ir}(111)$ : Physisorption with Chemical Modulation, Phys. Rev. Lett. 107, 036101 (2011).

[41] P. A. Khomyakov, G. Giovannetti, P. C. Rusu, G. Brocks, J. van den Brink, and P. J. Kelly, First-Principles Study of the Interaction and Charge Transfer between Graphene and Metals, Phys. Rev. B 79, 195425 (2009).

[42] S. Rusponi, M. Papagno, P. Moras, S. Vlaic, M. Etzkorn, P. M. Sheverdyaeva, D. Pacilé, H. Brune, and C. Carbone, Highly Anisotropic Dirac Cones in Epitaxial Graphene Modulated by an Island Superlattice, Phys. Rev. Lett. 105, 246803 (2010).

[43] C. Chen, J. Avila, and M. C. Asensio, Electronic Structure of Polycrystalline CVD-Graphene Revealed by NanoARPES, J. Phys. Conf. Ser. 849, 012019 (2017).

[44] X. Liu, C.Z. Wang, M. Hupalo, Y.X. Yao, M. C. Tringides, W. C. Lu, and K. M. Ho, Adsorption and Growth Morphology of Rare-Earth Metals on Graphene Studied by Ab Initio Calculations and Scanning Tunneling Microscopy, Phys. Rev. B 82, 245408 (2010).

[45] A. B. Shick and A. Denisov, Magnetism of 4f-Atoms Adsorbed on Metal and Graphene Substrates, J. Magn. Magn. Mater. 475, 211 (2019).

[46] L. Peters, I. Di Marco, P. Thunström, M. I. Katsnelson, A. Kirilyuk, and O. Eriksson, Treatment of $4 f$ States of the Rare Earths: The Case Study of TbN, Phys. Rev. B 89, 205109 (2014).

[47] P. K. Hansma, Inelastic Electron Tunneling, Phys. Rep. 30, 145 (1977).

[48] B. C. Stipe, M. A. Rezaei, and W. Ho, Single-Molecule Vibrational Spectroscopy and Microscopy, Science 280, 1732 (1998).

[49] H. Ibach, Physics of Surfaces and Interfaces (SpringerVerlag, Berlin, Heidelberg, 2006).

[50] S. Schumacher, T. O. Wehling, P. Lazić, S. Runte, D. F. Förster, C. Busse, M. Petrović, M. Kralj, S. Blügel, N. Atodiresei, V. Caciuc, and T. Michely, The Backside of Graphene: Manipulating Adsorption by Intercalation, Nano Lett. 13, 5013 (2013).

[51] J. Cui, J. D. White, and R. D. Diehl, Anomalous Inelastic He-Atom Scattering Intensities for the Vibrational Modes of Alkali Metals on Graphite, Surf. Sci. Lett. 293, L841 (1993).

[52] P. R. Forrester, T. Bilgeri, F. Patthey, H. Brune, and F. D. Natterer, Antiferromagnetic MnNi Tips for Spin-Polarized Scanning Probe Microscopy, Rev. Sci. Instrum. 89, 123706 (2018).

[53] See Supplemental Material at http://link.aps.org/ supplemental/10.1103/PhysRevX.10.031054 for Movie generated from a series of 59 STM images acquired consecutively on the region indicated by the dashed box in Fig. 3(a), enclosing one Ho and four Dy atoms $\left(V_{\mathrm{t}}=+2 \mathrm{mV}, I_{\mathrm{t}}=20 \mathrm{pA}, T=6.5 \mathrm{~K}\right)$. Each image was acquired in $1 \mathrm{~min} 48 \mathrm{~s}$.

[54] A. A. Khajetoorians, B. Baxevanis, C. Hübner, T. Schlenk, S. Krause, T. O. Wehling, S. Lounis, A. Lichtenstein, D. Pfannkuche, J. Wiebe, and R. Wiesendanger, 
Current-Driven Spin Dynamics of Artificially Constructed Quantum Magnets, Science 339, 55 (2013).

[55] R. Schmidt, C. Lazo, H. Hölscher, U. H. Pi, V. Caciuc, A. Schwarz, R. Wiesendanger, and S. Heinze, Probing the Magnetic Exchange Forces of Iron on the Atomic Scale, Nano Lett. 9, 200 (2009).

[56] S. Yan, D.-J. Choi, J. A. J. Burgess, S. Rolf-Pissarczyk, and S. Loth, Control of Quantum Magnets by Atomic Exchange Bias, Nat. Nanotechnol. 10, 40 (2015).

[57] J. Hermenau, M. Ternes, M. Steinbrecher, R. Wiesendanger, and J. Wiebe, Long Spin-Relaxation Times in a TransitionMetal Atom in Direct Contact to a Metal Substrate, Nano Lett. 18, 1978 (2018).

[58] D. Coffey, J. L. Diez-Ferrer, D. Serrate, M. Ciria, C. de la Fuente, and J. I. Arnaudas, Antiferromagnetic Spin Coupling between Rare Earth Adatoms and Iron Islands Probed by Spin-Polarized Tunneling, Sci. Rep. 5, 13709 (2015).

[59] M. Steinbrecher, A. Sonntag, M. dos Santos Dias, M. Bouhassoune, S. Lounis, J. Wiebe, R. Wiesendanger, and A. A. Khajetoorians, Absence of a Spin-Signature from a Single Ho Adatom as Probed by Spin-Sensitive Tunneling, Nat. Commun. 7, 10454 (2016).

[60] F. D. Natterer, F. Donati, F. Patthey, and H. Brune, Thermal and Magnetic-Field Stability of Holmium Single-Atom Magnets, Phys. Rev. Lett. 121, 027201 (2018).

[61] R. Wiesendanger, H.-J. Güntherodt, G. Güntherodt, R. J. Gambino, and R. Ruf, Observation of Vacuum Tunneling of Spin-Polarized Electrons with the Scanning Tunneling Microscope, Phys. Rev. Lett. 65, 247 (1990).

[62] W. Paul, K. Yang, S. Baumann, N. Romming, T. Choi, C. P. Lutz, and A. J. Heinrich, Control of the Millisecond Spin Lifetime of an Electrically Probed Atom, Nat. Phys. 13, 403 (2017).

[63] F. D. Natterer, K. Yang, W. Paul, P. Willke, T. Choi, T. Greber, A. J. Heinrich, and C. P. Lutz, Reading and Writing Single-Atom Magnets, Nature (London) 543, 226 (2017).

[64] S. Loth, S. Baumann, C. P. Lutz, D. M. Eigler, and A. J. Heinrich, Bistability in Atomic-Scale Antiferromagnets, Science 335, 196 (2012).

[65] S. Rusponi, N. Weiss, T. Cren, M. Epple, and H. Brune, High Tunnel Magnetoresistance in Spin-Polarized Scanning Tunneling Microscopy of Co Nanoparticles on Pt(111), Appl. Phys. Lett. 87, 162514 (2005).

[66] J. C. Slonczewski, Conductance and Exchange Coupling of Two Ferromagnets Separated by a Tunneling Barrier, Phys. Rev. B 39, 6995 (1989).

[67] S. Picozzi and A. J. Freeman, Polarization Reduction in Half-Metallic Heusler Alloys: The Effect of Point Defects and Interfaces with Semiconductors, J. Phys. Condens. Matter 19, 315215 (2007).

[68] A. A. Sapozhnik, C. Luo, H. Ryll, F. Radu, M. Jourdan, H. Zabel, and H.-J. Elmers, Experimental Determination of Exchange Constants in Antiferromagnetic $\mathrm{Mn}_{2} \mathrm{Au}$, Phys. Rev. B 97, 184416 (2018).

[69] F. Liu, D. S. Krylov, L. Spree, S. M. Avdoshenko, N. A. Samoylova, M. Rosenkranz, A. Kostanyan, T. Greber, A. U. B. Wolter, B. Büchner, and A. A. Popov, Single Molecule Magnet with an Unpaired Electron Trapped between Two Lanthanide Ions inside a Fullerene, Nat. Commun. 8, 16098 (2017).

[70] C. A. Gould, K. R. McClain, J. M. Yu, T. J. Groshens, F. Furche, B. G. Harvey, and J. R. Long, Synthesis and Magnetism of Neutral, Linear Metallocene Complexes of Terbium(II) and Dysprosium(II), J. Am. Chem. Soc. 141, 12967 (2019).

[71] V. Dubrovin, A. A. Popov, and S. Avdoshenko, Magnetism in Ln Molecular Systems with 4f/Valence-Shell Interplay (FV-Magnetism), Chem. Commun. (Cambridge) 55, 13963 (2019).

[72] F. Donati, S. Rusponi, S. Stepanow, C. Wäckerlin, A. Singha, L. Persichetti, R. Baltic, K. Diller, F. Patthey, E. Fernandes, J. Dreiser, Ž. Šljivančanin, K. Kummer, C. Nistor, P. Gambardella, and H. Brune, Science 352, 318 (2016).

[73] R. Gaisch, J. K. Gimzewski, B. Reihl, R. R. Schlittler, M. Tschudy, and W. D. Schneider, Low-Temperature UltraHigh Vacuum Scanning Tunneling Microscope, Ultramicroscopy 42-44, 1621 (1992).

[74] J. M. Wills, M. Alouani, P. Andersson, A. Delin, O. Eriksson, and O. Grechnyev, Full-Potential Electronic Structure Method, edited by H. Dreysse, Electronic Structure and Physical Properties of Solids: Springer Series in Solid-State Sciences (Springer-Verlag, Berlin, 2010).

[75] K. Lejaeghere et al., Reproducibility in Density Functional Theory Calculations of Solids, Science 351, aad3000 (2016).

[76] J. P. Perdew, K. Burke, and M. Ernzerhof, Generalized Gradient Approximation Made Simple, Phys. Rev. Lett. 77, 3865 (1996).

[77] A. Delin, L. Fast, B. Johansson, O. Eriksson, and J. M. Wills, Cohesive Properties of the Lanthanides: Effect of Generalized Gradient Corrections and Crystal Structure, Phys. Rev. B 58, 4345 (1998).

[78] L. Peters, I. Di Marco, M. S. Litsarev, A. Delin, M. I. Katsnelson, A. Kirilyuk, B. Johansson, B. Sanyal, and O. Eriksson, Valence and Spectral Properties of Rare-Earth Clusters, Phys. Rev. B 92, 035143 (2015).

[79] A. L. Kozub, A. B. Shick, F. Máca, J. Kolorenč, and A. I. Lichtenstein, Electronic Structure and Magnetism of Samarium and Neodymium Adatoms on Free-Standing Graphene, Phys. Rev. B 94, 125113 (2016).

[80] D. D. Koelling and B. N. Harmon, A Technique for Relativistic Spin-Polarised Calculations, J. Phys. C 10, 3107 (1977).

[81] G. Kotliar, S. Y. Savrasov, K. Haule, V. S. Oudovenko, O. Parcollet, and C. A. Marianetti, Electronic Structure Calculations with Dynamical Mean-Field Theory, Rev. Mod. Phys. 78, 865 (2006).

[82] P. Thunström, I. Di Marco, A. Grechnev, S. Lebègue, M. I. Katsnelson, A. Svane, and O. Eriksson, Multiplet Effects in the Electronic Structure of Intermediate-Valence Compounds, Phys. Rev. B 79, 165104 (2009).

[83] I. L. M. Locht, Y. O. Kvashnin, D. C. M. Rodrigues, M. Pereiro, A. Bergman, L. Bergqvist, A. I. Lichtenstein, M. I. Katsnelson, A. Delin, A. B. Klautau, B. Johansson, I. Di Marco, and O. Eriksson, Standard Model of the Rare Earths 
Analyzed from the Hubbard I Approximation, Phys. Rev. B 94, 085137 (2016).

[84] L. V. Pourovskii, B. Amadon, S. Biermann, and A. Georges, Self-Consistency over the Charge Density in Dynamical Mean-Field Theory: A Linear Muffin-Tin Implementation and Some Physical Implications, Phys. Rev. B 76, 235101 (2007).

[85] K. Haule, Exact Double Counting in Combining the Dynamical Mean Field Theory and the Density Functional Theory, Phys. Rev. Lett. 115, 196403 (2015).

[86] O. Grånäs, I. Di Marco, P. Thunström, L. Nordström, O. Eriksson, T. Björkman, and J. M. Wills, Charge Self-Consistent Dynamical Mean-Field Theory Based on the Full-Potential Linear Muffin-Tin Orbital Method:
Methodology and Applications, Comput. Mater. Sci. 55, 295 (2012).

[87] M. Brooks, Thomas-Fermi Screening of Exchange Interactions, J. Phys. Condens. Matter 13, L469 (2001).

[88] F. Bultmark, F. Cricchio, O. Grånäs, and L. Nordström, Multipole Decomposition of $\mathrm{LDA}+U$ Energy and Its Application to Actinide Compounds, Phys. Rev. B 80, 035121 (2009).

[89] F. D. Natterer, F. Patthey, and H. Brune, Quantifying Residual Hydrogen Adsorption in Low-Temperature STMs, Surf. Sci. 615, 80 (2013).

[90] F. Donati, Q. Dubout, G. Autès, F. Patthey, F. Calleja, P. Gambardella, O. V. Yazyev, and H. Brune, Magnetic Moment and Anisotropy of Individual Co Atoms on Graphene, Phys. Rev. Lett. 111, 236801 (2013). 